ISSN-PRINT 1794-9831 / E-ISSN 2322-7028

Vol. 18 No 2 / Mayo - Agosto, 2021 / Cúcuta, Colombia.

doi) https://doi.org/10.224663/17949831.2872

Revisión

\title{
Perfil del estilo de vida en estudiantes de una Universidad Pública
}

\author{
Lifestyle profile of Public University students \\ Perfil do estilo de vida em estudantes de uma Universidade Pública
}

\author{
Sergio Charry-Méndez ${ }^{1}$ \\ Esperanza Cabrera-Díaz ${ }^{2}$
}

\section{Resumen}

Objetivo: Determinar los comportamientos del estilo de vida de los estudiantes de primer semestre de una Universidad Pública. Materiales y método: Investigación cuantitativa, descriptiva de corte trasversal. El tamaño de la muestra fue de 113 estudiantes matriculados para el periodo académico 2020-1. Se utilizó un instrumento tipo encuesta estructurada elaborado por el proyecto Institucional Universidad Saludable. Los datos se trabajaron a través de Excel y el programa SPSS versión 22, mediante análisis estadístico descriptivo univariado de frecuencias y tablas de contingencia. Resultados: la mayoría estudiantes no realiza ejercicio físico $70 \%$, el $51 \%$ no realiza ningún tipo de actividad física, El consumo de tabaco es alto y más de la mitad son consumidores habituales de alcohol, más de la mitad presenta angustia y casi la mitad ansiedad. La calidad de la dieta la mitad la consideran mala y hay bajo consumo de frutas. El consumo de sal añadida a los alimentos es referido por la mayoría $82 \%$ de los estudiantes. El $28 \%$ presenta sobrepeso y obesidad. La mayoría desconoce sus niveles de colesterol y triglicéridos. No existe en casi la mitad redes de apoyo entre compañeros y familiares. Conclusiones: Se encontró que el perfil de estilo de vida de los estudiantes de primer semestre de una universidad pública se caracteriza por el sedentarismo, el consumo habitual de cerveza y cigarrillo, la mala alimentación, el sobrepeso y la obesidad, la ansiedad y angustia.

Palabras claves: Promoción de la salud, estilo de vida, Enfermedad crónica, Factores de riesgo.

\footnotetext{
Abstract

Objective: Determine the lifestyle behaviors of first semester Public University Students. Materials and method: Quantitative, descriptive, cross-sectional research. The size of the sample consisted of 113 enrolled students for the 2020-1 semester. The instrument of structured interview made by the Institutional Project Universidad Saludable was used. The data were worked using Excel and SPSS version 22, through an univariate descriptive statistical analysis of frequencies and contingency tables. Results: Most of the students do not do physical exercise $70 \%, 51 \%$ do not do any physical activity, there is a high consumption of tobacco and more than half are frequent consumers of alcohol, more than half show anguish and almost half show anxiety. Half of the students consider that the quality of their diet is bad and there is a low consumption of fruits. The consumption of added salt to food is mentioned by most students $82 \%$. $28 \%$ are overweight or obese. Most do not know their levels of cholester$\mathrm{ol}$ and triglycerides. There are no support networks of schoolmates and family in almost half of the students. Conclusions: It was found that the lifestyle profile of first semester students
}

\begin{abstract}
Autor de correspondencia*
1* Psicólogo Universidad Surcolombiana. Psicólogo proyecto institucional Usco Saludable. Universidad Surcolombiana. Neiva, Huila. Correo: sergio. charry@usco.edu.co (D) 0000-00020949-3791

2 Psicóloga Universidad Javeriana. Magister en Educación y Desarrollo Comunitario. Especialización y Doctorado en Bioética. Docente Universidad Surcolombiana. Coordinadora del Proyecto Universidad Saludable. Directora del Departamento de Medicina Social, Programa de Medicina. Facultad de Salud. Neiva -Huila. Correo: cabreraesperanza@usco.edu.co (iD) 00000001-8868-2832
\end{abstract}

Recibido: 24 febrero 2021

Aprobado: 26 abril 2021

Para citar este artículo / To reference this article / Para citar este artigo:

Charry-Mendez S, Cabrera-Díaz E. Perfil del estilo de vida en estudiantes de una Universidad Pública. Rev. cienc. cuidad. 2021; 18(2):82-95. https://doi. org/10.22463/17949831.2872

(C) Universidad Francisco de Paula Santander. Este es un artículo bajo la licencia CC-BY-NC-ND

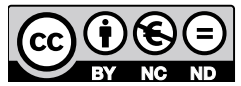




\section{Scientific Journal of Nursing}

in a public university is characterized by sedentary lifestyle, frequent consumption of beer and cigarettes, bad nutrition, overweight and obesity, anguish, and anxiety.

Keywords: Health promotion, lifestyle, Chronic disease, Risk factors.

\section{Resumo}

Objetivo: Determinar o comportamento do estilo de vida dos alunos de primeiro semestre de uma Universidade Pública. Materiais e métodos: Pesquisa quantitativa, descritiva, transversal. O tamanho da amostra foi de 113 alunos matriculados no período acadêmico 2020-1. Utilizou-se um formulário estruturado criado pelo projeto institucional Universidade saudável. Os dados foram processados no Excel e no programa SPSS versão 22, realizando-se uma analise univariada de frequência e tabelas de contingência. Resultados: A maioria dos alunos não faz exercício físico ( $70 \%$ ), o $51 \%$ não realiza nenhum tipo de atividade física. O consumo de tabaco é alto e mais da metade são consumidores regulares de álcool, mais da metade presenta angustia e quase a metade ansiedade. A qualidade da dieta, a metade a consideram ruim com baixo consumo de frutas. O consumo de sal adicionada aos alimentos é referida pelo $82 \%$ dos alunos. $28 \%$ presenta sobrepeso e obesidade. A maioria desconhece os seus níveis de colesterol e triglicerídeos. Em quase a metade deles não existe rede de apoio entre colegas ou familiares. Conclusões: Encontrou-se que o perfil do estilo de vida dos alunos de primeiro período de uma Universidade Pública caracteriza-se pelo sedentarismo, o consumo regular de cerveja e cigarro, a má alimentação, o sobrepeso e obesidade, a ansiedade e angustia.

Palavras-chave: Promoção da saúde, estilo de visa, doença crônica, fatores de risco.

\section{Introducción}

Los estilos de vida saludables pueden entenderse como un modelo de conducta asociados con la salud, definidos por las decisiones que toman las personas en sus interacciones sociales, socioeconómicas y ambientales (1). Igualmente, los estilos de vida saludables son un conjunto de comportamientos que se realizan de forma estable (como por ejemplo hacer actividad física todos los días) posibilitados o limitados por la condición social.

Conviene señalar que la conversión epidemiológica del modelo de enfermedad pasó de un patrón agudo a uno crónico, donde tienen importancia los factores ambientales y los estilos de vida de las personas. En este sentido, cobra relevancia el debate respecto a los estilos de vida saludable y su impacto sobre el estado de la salud. Los estilos de vida en el mundo occidental suelen manifestarse por comportamientos que se establecen como factores de riesgo para la salud, tales como: el consumo de tabaco, las dietas malsanas, la inactividad física y el uso nocivo del alcohol; estos elementos representan una idea clara de comportamientos perjudiciales o de riesgo. Todas estas conductas están íntimamente asociadas a factores de riesgo que incrementan la posibilidad de padecer enfermedades no trasmisibles (ENT) las cuales son la principal causa de muerte en todo el mundo (2). Las ENT son patologías que juegan un papel importante en la mortalidad de la población mediante sucesos que se originaron años atrás; es decir, son enfermedades de larga duración y de evolución lenta (Enfermedad cardiovascular, diabetes, obesidad, hipertensión arterial, sedentarismo y dieta inadecuada) por tanto, se constituyen en uno de los mayores retos para el sistema de salud en los cinco continentes.

Las ENT ocasionan dos de cada tres muertes en la población general de América Latina y casi la mitad de todas las defunciones en personas menores de 70 años. Las enfermedades no transmisibles (ENT), son la causa de defunción más importante en el mundo. Las enfermedades cardio y cerebrovasculares (ECV) son las primeras causas de muerte y discapacidad, lo que representan un $30 \%$ de todas las muertes registradas en el mundo (3,4). En Colombia entre 2005 y 2014, las enfermedades del sistema circulatorio representaron 


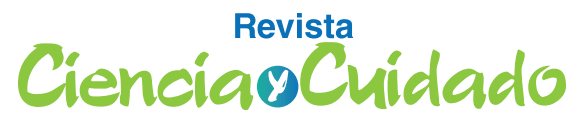

Scientific Journal of Nursing

la causa de muerte número uno en hombres y mujeres, seguidas de las enfermedades cerebrovasculares y en tercer lugar las hipertensivas. Éstas comparten factores de riesgo comunes como el tabaquismo, la inactividad física, el estrés, el uso nocivo del alcohol y la dieta no saludable (5).

Se cree que gran parte de las enfermedades que afectan a la población en el mundo se pueden prevenir a través del establecimiento y modificación de estilos de vida saludable. La universidad y centros educativos son espacios fundamentales para la conformación de estilo y hábitos de vida saludable, debido sobre todo a la influencia que ejercen en los jóvenes, así como el tiempo que permanecen en estos, lo que requiere un trabajo multidisciplinario dirigido a modificar de forma positiva los factores de riesgo, que subyacen en el estilo de vida actual de esta población.

La transición a la juventud conlleva consigo cambios físicos, psicológicos y sociales, los cuales cambian la forma de vida de los jóvenes y los estimula a realizar un papel activo en su propia salud y autocuidado, responsabilidad que antes era de sus padres. En esta etapa buscan su independencia, así como el querer impresionar a los demás y desafiar los propios límites. Este proceder hace que participen de comportamientos riesgosos, que a su vez son determinantes en el tipo de morbilidad, la discapacidad y la mortalidad en su edad adulta. Lo anterior, se acentúa con el ingreso a la universidad y la adaptación a este tipo de vida. Se calcula que aproximadamente dos de cada tres muertes prematuras y un tercio de la carga total de morbilidad en los adultos están relacionadas con situaciones o comportamientos que se inician durante la adolescencia (6). Además, el estado de salud de los jóvenes incide en su porvenir, pues termina afectando la salud de sus propios hijos; así como la repercusión sobre los altos costos de atención, años de vida potencialmente perdidos e incluso el detrimento en la calidad de vida y en el desempeño social (6).

Este documento da cuenta de un estudio elaborado sobre estudiantes de una Universidad Pública de Colombia, con el propósito de determinar el perfil de estilo de vida que presentan al ingresar a la universidad. Con los resultados que se obtengan se pretende generar procesos que favorezcan el establecimiento de un estilo de vida saludable.

\section{Objetivos}

\section{Objetivo general}

Determinar los comportamientos del estilo de vida de los estudiantes de primer semestre de una Universidad Pública.

\section{Objetivos específicos}

- Establecer las características sociodemográficas de la población

- Definir la actividad física semanal que practican los estudiantes de la universidad.

- Establecer la prevalencia de sobrepeso y obesidad según IMC de los estudiantes.

- Identificar el consumo de cigarrillo y cerveza en los estudiantes de primer semestre de la Universidad Surcolombiana.

- Valorar la presencia de estresores en los estudiantes.

- Describir el tipo de alimentación de los estudiantes.

- Identificar la responsabilidad en salud que presentan los estudiantes.

- Establecer la red de relaciones sociales de los estudiantes.

\section{Materiales y métodos}

Se realizó un estudio descriptivo de corte trasversal, con los estudiantes del primer semestre de los programas de la Facultad de Educación de toda la cohorte 2020-1 para un total de 113 estudiantes. Respecto al cálculo de la muestra se utilizó el programa EPIDAT versión 4.2 con un tamaño de población de 1582 estudiantes y una prevalencia del 16,63\%, un nivel de confianza del 95\% y precisión del 7,6\%, obteniendo como mínimo una muestra conformada por 113 estudiantes. La selección de los individuos se desarrolló mediante un muestro aleatorio simple, a partir de las listas entregadas por el sistema de verificación de participación en las actividades del Proyecto Institucional Usco Saludable.

Además, se elaboró un instrumento tipo encuesta auto aplicable de selección. En la primera fase de la metodología se realizó una revisión de la literatura, para conformar la definición del constructo de la encuesta, tomando como punto de partida los niveles de consumo y actividades propuestas por la Organización Mundial de la Salud (OMS), en función de: señales de dependencia, 
frecuencia y problemas asociados al consumo excesivo de algunos alimentos o sustancias. Una vez identificados los indicadores para cada uno de los constructos se realizó la operacionalización de las variables y se elaboró el instrumento.

Se debe agregar que la encuesta implementada para la recolección de la información está presentada en un solo bloque, conformado por 15 ítems y preguntas que hacen referencia a: datos sociodemográficos, ejercicio físico y actividad física, conocimiento acerca del estado de salud (índice de masa corporal, perímetro abdominal, cigarrillo/día, consumo de alcohol/semana, autoimagen del peso); hábitos alimentarios (percepción dietaría, porciones de fruta/día, consumo de frituras/semana, consumo de sal); antecedentes tóxicos; estado de angustia y ansiedad en el último mes; y, frecuencia con la que comparte tiempo con pares o amigos cercanos. La intención fue crear un instrumento que permitiera identificar los factores de riesgo en el estilo de vida de los estudiantes de la universidad. Para ello este cuestionario presenta una serie de afirmaciones sobre los hábitos de vida actuales; por tanto, no hay respuesta correcta o incorrecta. En definitiva, lo que se le pide al estudiante es que responda espontáneamente la opción que refleje mejor sus hábitos. Para esto, cada participante contestó en una escala tipo Likert, describiendo la frecuencia, cantidad y conocimiento de cada interrogante.

Hay que precisar que la encuesta se sometió a revisión por parte de expertos en el área de estilos y hábitos de vida saludable. Una vez realizada la revisión por expertos y efectuar las sugerencias por estos, se estableció la validez de contenido mediante el coeficiente Kappa de Fleiss. Según los resultados de los datos promedio del índice Kappa de Fleiss $(\mathrm{k}=0,61)$ la concordancia o nivel de acuerdo interjueces del instrumento es bueno, o sea, que los jueces están de acuerdo en que los 15 ítems y sus respectivas escalas presentaron significativamente cada una de las dimensiones, referidas a: suficiencia, claridad, coherencia y relevancia. La fiabilidad se calculó con el índice Alfa de Cronbach con un resultado de 0,802 , que indicó una aceptable consistencia interna del instrumento. Por tanto, el cuestionario fue óptimo para identificar el perfil de estilo de vida de los estudiantes universitarios (7). La información se recolectó en el centro social los Cerros en donde se desarrollaba la Jornada Valórate Usco del Proyecto Institucional Usco saludable, con una duración de 15 minutos aproximadamente.

También, se realizó un análisis estadístico descrip- tivo univariado de frecuencias mediante el software SPSS ${ }^{\circledR}$ versión 22. Además, se elaboraron tablas de contingencia para hallar la fuerza de asociación entre las variables. Igualmente, se estableció un nivel de significancia estadística del 0,05 $(\mathrm{p}<0,05)$. Del mismo modo, se contó con la participación voluntaria y el consentimiento informado de cada estudiante. En el caso de los menores de edad, el consentimiento fue firmado por los padres con el asentimiento informado de cada estudiante. Para las variables antropométricas se contó con una báscula digital TZ referencia $5687 \mathrm{~F}$ y se empleó una cinta métrica tipo tallímetro, modelo Seca referencia 1337201.

Conviene subrayar, que como la investigación se desarrolló con estudiantes universitarios, se obtuvo el consentimiento informado teniendo en cuenta los principios de confidencialidad, el anonimato y los demás suscrito en: la Declaración de Helsinki; el código de Núremberg y la Resolución 8430 de 1993 del Ministerio de Salud de Colombia y las Normas de Buenas Prácticas Clínicas; y, las Pautas Éticas Internacionales para la Experimentación Biomédica en Seres Humanos. Además, se contó con el aval del Comité de Ética y directivas de la Universidad Surcolombiana, acuerdo 056 del 2019 PDI

\section{Resultados}

El $54 \%$ de la población es mayor de edad, el $60 \%$ corresponde al sexo femenino. El $70 \%$ de los estudiantes nunca hace ejercicio físico y el 51\% no realiza ningún tipo de actividad física. En cuanto a las mediciones antropométricas, el $28 \%$ tiene sobrepeso y obesidad; en relación con la percepción del peso, el 44\% indicó que tenía un peso adecuado. Con respecto a los antecedentes tóxicos, el 22\% dice que fuma entre 6 y 15 cigarrillos diarios. El 58\% afirma consumir entre 7 y 14 cervezas semanales. En relación a la variable de ansiedad, el $49 \%$ dijo que casi siempre estaba ansioso y el $53 \%$ manifestó estar casi siempre angustiado. Con relación al consumo de porciones de frutas por día, solo el $2 \%$ consume más de cinco porciones al día, el 30\% consume entre cinco o más alimentos fritos a la semana. En el consumo de sal, el $82 \%$ asevera que agrega sal a los alimentos. Solo el 7\% de los estudiantes considera que su dieta es muy buena. El 85\% de los universitarios dijo no conocer su grado de colesterol y el $80 \%$ no conoce su grado de triglicéridos. En la red social el $41 \%$ manifestó que nunca comparte tiempo con amigos cercanos y familiares (Ver Tabla 1).

Tabla 1. Descriptivo del estilos de vida presente en estudiantes 
Scientific Journal of Nursing

universitarios de primer semestre.

\begin{tabular}{|c|c|c|c|}
\hline \multicolumn{2}{|c|}{ VARIABLE } & FRECUENCIA & PORCENTAJE \\
\hline \multicolumn{4}{|c|}{ Variable sociodemográfica } \\
\hline \multirow[t]{2}{*}{ Sexo } & $\mathrm{F}$ & 68 & $60 \%$ \\
\hline & M & 45 & $40 \%$ \\
\hline \multirow[t]{2}{*}{ Edad } & $<18^{\mathrm{a}}$ & 52 & $46 \%$ \\
\hline & $>18^{\mathrm{a}}$ & 61 & $54 \%$ \\
\hline \multicolumn{4}{|c|}{ Variable actividad física } \\
\hline \multirow[t]{4}{*}{ Ejercicio físico } & Nunca & 79 & $70 \%$ \\
\hline & Una vez por semana & 14 & $12 \%$ \\
\hline & $2-3$ veces por semana & 15 & $13 \%$ \\
\hline & Casi todos los días & 5 & $4 \%$ \\
\hline \multirow[t]{4}{*}{ Actividad física } & Nunca & 58 & $51 \%$ \\
\hline & Una vez por semana & 32 & $28 \%$ \\
\hline & $2-3$ veces por semana & 15 & $13 \%$ \\
\hline & Casi todos los días & 9 & $8 \%$ \\
\hline \multicolumn{4}{|c|}{ Variable IMC } \\
\hline \multirow[t]{4}{*}{ IMC } & $<18,5$ infrapeso & 23 & $20 \%$ \\
\hline & $18,5-24,9$ normal & 58 & $51 \%$ \\
\hline & 25-29,9 sobrepeso & 18 & $16 \%$ \\
\hline & 30 y más obesidad & 14 & $12 \%$ \\
\hline \multirow[t]{4}{*}{ Percepción de Peso } & Adecuado & 50 & $44 \%$ \\
\hline & Por debajo de mi pesado adecuado & 30 & $27 \%$ \\
\hline & Por encima de mi peso adecuado & 27 & $24 \%$ \\
\hline & Con exceso de peso & 6 & $5 \%$ \\
\hline \multicolumn{4}{|c|}{ Variable antecedentes tóxicos } \\
\hline \multirow[t]{4}{*}{ Consumo de cigarrillo diario } & No fumo & 12 & $11 \%$ \\
\hline & Menos de 5 cigarrillos diarios & 65 & $58 \%$ \\
\hline & Entre 6 a 15 cigarrillos diarios & 25 & $22 \%$ \\
\hline & Fumo 16 cigarrillos diarios & 11 & $10 \%$ \\
\hline \multirow{4}{*}{$\begin{array}{l}\text { Consumo de cerveza a la se- } \\
\text { mana }\end{array}$} & Nunca consumo & 7 & $6 \%$ \\
\hline & Entre 3 y 6 cervezas & 25 & $22 \%$ \\
\hline & Entre 7 y 14 cervezas & 65 & $58 \%$ \\
\hline & Más de 15 cervezas & 16 & $14 \%$ \\
\hline \multicolumn{4}{|c|}{ Variable estressores } \\
\hline \multirow[t]{4}{*}{ Angustia último mes } & Nunca & 12 & $11 \%$ \\
\hline & Algunas veces & 21 & $19 \%$ \\
\hline & Siempre & 20 & $18 \%$ \\
\hline & Casi siempre & 60 & $53 \%$ \\
\hline
\end{tabular}


Scientific Journal of Nursing

\begin{tabular}{|c|c|c|c|}
\hline \multirow[t]{4}{*}{ Ansiedad último mes } & Nunca & 4 & $4 \%$ \\
\hline & Algunas veces & 30 & $27 \%$ \\
\hline & Siempre & 24 & $21 \%$ \\
\hline & Casi siempre & 55 & $49 \%$ \\
\hline \multicolumn{4}{|c|}{ Variables alimentación sana } \\
\hline \multirow[t]{4}{*}{ Consumo de frutas } & Ninguna porción & 48 & $42 \%$ \\
\hline & 1 y 2 porción & 35 & $31 \%$ \\
\hline & 3 y 4 porciones & 28 & $25 \%$ \\
\hline & 5 o más porciones & 5 & $2 \%$ \\
\hline \multirow{4}{*}{$\begin{array}{l}\text { Consumo de alimentos fritos a } \\
\text { La semana }\end{array}$} & Nunca & 16 & $14 \%$ \\
\hline & Entre 1 y 2 & 23 & $20 \%$ \\
\hline & Entre $3 y 4$ & 40 & $35 \%$ \\
\hline & 5 o mas & 34 & $30 \%$ \\
\hline \multirow[t]{4}{*}{ Sal que añade a las comidas } & Ninguna & 20 & $18 \%$ \\
\hline & Media cucharada de sal & 37 & $33 \%$ \\
\hline & Una cucharada de sal & 22 & $19 \%$ \\
\hline & Dos o más cucharadas de sal & 34 & $30 \%$ \\
\hline \multirow[t]{4}{*}{ Alimentación diária } & Mala & 56 & $50 \%$ \\
\hline & Regular & 34 & $30 \%$ \\
\hline & Buena & 15 & $13 \%$ \\
\hline & Muy buena & 8 & $7 \%$ \\
\hline \multicolumn{4}{|c|}{ Variable responsabilidad en Salud } \\
\hline \multirow[t]{4}{*}{ Conocimiento de colesterol } & Nunca & 96 & $85 \%$ \\
\hline & Algunas veces & 14 & $12 \%$ \\
\hline & Siempre & 2 & $2 \%$ \\
\hline & Casi siempre & 1 & $1 \%$ \\
\hline \multirow[t]{4}{*}{ Conocimiento de Triglicéridos } & Nunca & 90 & $80 \%$ \\
\hline & Algunas veces & 19 & $17 \%$ \\
\hline & Siempre & 2 & $2 \%$ \\
\hline & Casi siempre & 2 & $1 \%$ \\
\hline \multicolumn{4}{|c|}{ Variable red social } \\
\hline \multirow{4}{*}{$\begin{array}{l}\text { Tiempo con amigos cercanos, } \\
\text { familia o compañeros }\end{array}$} & Nunca & 46 & $41 \%$ \\
\hline & Algunas veces & 38 & $34 \%$ \\
\hline & Siempre & 12 & $11 \%$ \\
\hline & Casi siempre & 17 & $15 \%$ \\
\hline
\end{tabular}

Fuente: Elaboración propia.

En el análisis inferencial se encontró relación significa- lino más activo físicamente que el femenino, en cuanto tiva entre sexo y ejercicio físico, siendo el sexo mascu- al consumo de cerveza este se presenta en menor grado 


\section{Scientific Journal of Nursing}

en el sexo masculino (Tabla 2).

Tabla 2. Variables y nivel de significancia

\begin{tabular}{cc}
\hline Variables & Nivel de Significancia \\
\hline Sexo Masculino / ejercicio físico & 0,000
\end{tabular}

Sexo Masculino/ consumo de cerveza

Fuente: Elaboración propia.

\section{Discusión}

Los resultados obtenidos en la presente investigación evidencian que los estudiantes universitarios no realizan ejercicio físico, según lo recomendado por la OMS. Esto demuestra el alto grado de sedentarismo presente en esta etapa de la vida. Situaciones semejantes reportan otras investigaciones, con bajos niveles de ejercicio físico entre universitarios $(8,9)$ en donde hay reducción de actividades recreativas y deportivas, debido a la transición de la adolescencia a la adultez. Se suma a esto el que los estudiantes aseguran no hacer ejercicio por: falta de tiempo, cansancio, pereza y la falta de interés por el deporte y la actividad física (10). Sobre este aspecto, el presente estudio, evidencia que existe diferencia según sexo en lo referente a la práctica del ejercicio físico, encontrándose que las mujeres son más sedentarias que los hombres. Este resultado coincide con lo reportado en el estudio de Leyva et al., (11) quienes encontraron que las mujeres son significativamente más inactivas que los hombres. En este sentido, el ejercicio físico está influenciado por los roles de género que crean estereotipos los cuales intervienen en los intereses y motivaciones hacia la práctica deportiva. Al respecto los hombres están más motivados por los deportes, en cambio las mujeres prefieren actividades sociales y estéticas.

En lo relacionado a la actividad física la mitad de los estudiantes no la realiza; es decir, que son sedentarios. Los jóvenes universitarios dedican el mayor tiempo del día a las clases con poco espacio para realizar actividad física (12). Resultados similares se han encontrado en otros estudios nacionales e internacionales $(12,13)$, en los que se perciben bajas frecuencias en la realización de actividad física, constituyendo un grave riesgo de salud, que, con el tiempo, derivará en enfermedades crónicas no trasmisibles. Es importante motivar al estudiante sobre la necesidad de realizar ejercicio y actividad físicas, concientizándolo en la responsabilidad para el autocuidado de la salud, con el fin de mejorar su ca- lidad de vid,o0(14). La actividad física se constituye en una estrategia y herramienta efectiva que beneficia la salud a nivel biológico, psicosocial y cognitivo $(12,14)$. De ahí, que se deba fomentar modalidades de actividad física que sean de interés para los estudiantes, preferiblemente grupales, dado que el acompañamiento mejora la motivación y el disfrute de la actividad física (12).

Más de la mitad de los estudiantes presentaron un IMC dentro de límites normales, que coincide con los resultados encontrados en el estudio de Ruiz et al., (15). Sin embargo, el $28 \%$ presenta sobrepeso y obesidad, lo que concuerda con otras investigaciones $(16,17)$ en donde se ha encontrado un alto porcentaje de estudiantes con problemas nutricionales, por exceso en el IMC, debido a los inadecuados hábitos basados en el consumo de azúcares, harinas y grasas (18); a los componentes anteriores se suma la inactividad física (19). Por otro lado, el sobrepeso y la obesidad son factores que contribuyen al aumento de enfermedades degenerativas que perturban el desarrollo psicosocial y la calidad de vida (20). Los estudiantes con un estado nutricional inadecuado pueden verse afectados por aspectos psicológicos negativos, que afectan el rendimiento académico (21).

En cuanto a la percepción del peso corporal, casi la mitad de los estudiantes percibieron su peso adecuado (44\%), lo cual no concuerda con otros estudios (22) en donde se deduce que más de la mitad $(56 \%)$ de los estudiantes subestima o sobreestima su peso. Esta conducta puede ocasionar en el escolar exceso o restricciones alimentarias que intervienen en el estado nutricional (23).

Otro rasgo a tener en cuenta, es que casi la totalidad de los estudiantes manifiestan fumar a diario, esto no coincide con otras investigaciones donde se demuestra que una pequeña proporción de los estudiantes universitarios reconoció consumir tabaco $(24,25)$. En contraste, otros estudios $(26,27)$ reportan el alto consumo de tabaco entre universitarios., diferentes investigaciones indican que los estudiantes universitarios fuman por placer y para disminuir el estrés, la tensión, la angustia, la depresión y el nerviosismo generado por su actividad o por el cansancio (28). 
Llama la atención, el que prevalezca el consumo de alcohol por los jóvenes en Colombia $(29,30)$; pero además según algunos estudios este problema está relacionad con el delito (30). En la presente investigación de identificó que el 94\% de los estudiantes consume alcohol a la semana, lo que reafirma la problemática a nivel nacional, observando una mayor incidencia en hombres que en mujeres (p 0,007). El ambiente universitario se presta para el consumo de alcohol, conduciendo a los afectados a la deserción escolar, al bajo rendimiento académico, al ausentismo y a las dificultades académicas, familiares y sociales (31). Se ha encontrado que las razones más comunes por las que los jóvenes consumen alcohol son: la sensación de placer, tranquilidad o exaltación, la aprobación por parte de los pares, sentirse mejor o suplir carencias a nivel social, afectivo o intelectual (32).

Al mismo tiempo, el estudio mostró que casi la mitad de los estudiantes experimentan ansiedad casi siempre. El ingreso a la universidad trae consigo cambios significativos que alteran su cotidianidad propiciando un ritmo de vida acelerado que induce a la ansiedad $(33,34)$. Entre los factores académicos relacionados con la ansiedad están: la insatisfacción con la carrera, el desarraigo escolar, dificultades económicas, cargas familiares y laborales, percepción de menor apoyo social, disminución de la red social, dificultades en las relaciones con compañeros y docentes; así como el estrés generado por las responsabilidades académicas (sobrecarga académica, falta de tiempo para cumplir con obligaciones académicas y la realización de parciales) $(35,36)$.

También el estudio determina que más de la mitad de los estudiantes ha vivido la angustia en su vida universitaria. Esto coincide con otros autores quienes afirman que los niveles de angustia aumentan cuando se acercan las semanas de parciales y entrega de trabajos. Es importante destacar que la angustia afecta el rendimiento del estudiante, porque da origen a los lapsus mentales o a la confusión de las respuestas, disminuyendo su rendimiento en los parciales y evaluaciones (37). Ante esta situación, las estrategias que se deben implementar para el manejo de la ansiedad y la angustia hay que orientarlas hacia la mejora en los hábitos de estudio, alimentación saludable, actividad física, manejo adecuado del tiempo libre y la importancia del sueño/descanso, porque la respuesta al estrés depende de la disposición de cada uno para hacer frente a las situaciones.

Según este estudio, solo el 7\% de los estudiantes manifestaron que la alimentación que consumen diariamente es de muy buena calidad. Esto se relaciona con otras investigaciones $(38,39)$ en donde se demostró que la alimentación diaria de los estudiantes universitarios no cumplen con los aportes necesarios para la edad y la actividad que desarrollan (40). Según expertos y diferentes estudios $(41,38,39)$ los jóvenes prefieren comer mal y no modifican su alimentación por una más saludable, tampoco se acogen a estos cambios debido a la carga académica, que no les deja mucho tiempo para planificar esta actividad. Hay que precisar que el tipo de alimentación depende también de la oferta interna que ofrece la universidad, así como del precio de los diferentes productos. En función de las limitaciones anteriores los lleva a seleccionar la oferta externa alrededor de la universidad, en donde los alimentos se caracterizan por contener altos grados de grasas saturadas, pero además son más asequibles por los bajos precios. Se suma a lo anterior los inadecuados hábitos alimentarios que no les permite consumir merienda, no desayunan, lo que los obliga a ayunar por largas horas y a preferir comidas rápidas; pero tampoco realizan actividades físicas.

En todo caso, los estudiantes necesitan balancear el trabajo académico con sus necesidades nutricionales para mantener su bienestar. Es desde esta perspectiva que se les debe ofrecer productos nuevos, saludables y económicos en las cafeterías y restaurantes universitarios. De igual forma, hay que brindarles talleres sobre hábitos de estudio, manejo del presupuesto, hábitos de sueño, manejo del estrés y la ansiedad, así como organización del tiempo y los ingresos, de tal manera que sus compromisos académicos no afecten su salud. Es necesario recalcar que los talleres de educación alimentaria propician en los universitarios la importancia de la alimentación completa diaria, les permite reflexionar sobre el consumo de alimentos adecuados para prevenir enfermedades, evitando las grasas saturadas y aumentando el consumo de frutas y verduras, conforme a la pirámide nutricional y el plato del buen comer (42).

Con relación al consumo de frutas por los estudiantes, este estudio demostró que un porcentaje muy bajo consume las cinco porciones de fruta al día recomendadas por la OMS (43) y que casi la totalidad no las consumen o apenas sólo una a dos porciones diarias. El bajo consumo de frutas y verduras es un factor de riesgo causante de enfermedades cardiovasculares y algunos tipos de cáncer (44). El bajo consumo de frutas es un hábito alimentario inadecuado, paralelo al consumo excesivo de alcohol e inactividad física (44). De igual manera, el poco consumo de frutas está relacionado con la inaccesibilidad a este grupo de alimentos por cuestiones económicas. Es por eso, que se consume mayor cantidad de energía por la ingesta de otros alimentos y la poca efectividad en la oferta de frutas en los sitios donde los 


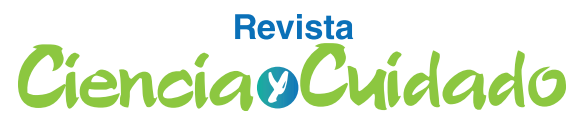

Scientific Journal of Nursing

jóvenes pasan la mayoría del tiempo $(45,46)$. Definitivamente es necesario fomentar en los estudiantes universitarios el consumo de frutas para mantener la salud y protegerse contra enfermedades crónicas no trasmisibles. De ahí la necesidad de concientizarlos como agentes responsables del autocuidado de su salud; pero además motivarlos para que sean futuros promotores y multiplicadores de estilos de vida saludable, que son vitales para el cuidado de su salud.

Por otro lado, el estudio muestra que el $30 \%$ de los estudiantes universitarios consumen cinco o más porciones de alimentos fritos a la semana, situación que coincide con otros estudios (45). Estos alimentos son de fácil adquisición y de consumo rápido, además están disponibles en cualquier momento, dado el contexto cultural. El deterioro del alimento se produce durante el proceso de preparación, cuando al freírse se destruyen los componentes nutricionales y se forman compuestos tóxicos que potencian el sabor y producen adicción, alterando el apetito.

Se debe agregar que la investigación evidenció que más del $80 \%$ de los estudiantes agregan sal a los alimentos. El $16.7 \%$ de los colombianos añaden más sal a sus alimentos después de servidos en la mesa, siendo el grupo de 19 a 30 años el que realiza esta práctica con mayor frecuencia, seguido por los de 14 a 18 años y los niños de 5 a 8 años (47). Se ha demostrado que existe una relación directa entre el consumo de sal y la hipertensión arterial, razón por la cual es necesario reducir la ingesta excesiva de sal en la dieta, con el fin de reducir las enfermedades cardiovasculares.

Igualmente se concluyó que existe desconocimiento sobre los niveles del colesterol en la sangre por parte del $85 \%$ de los estudiantes. Este dato es preocupante, puesto que el colesterol alto es un factor de riesgo que altera el organismo, mucho antes de que aparezcan síntomas o signos clínicos (48). Por esto, es necesario realizar exámenes regularmente después de los 20 años de edad, para identificar precozmente alguna alteración y poder intervenir a tiempo. Según la OMS, el colesterol alto es uno de los factores de riesgo de mortalidad en el mundo (49), siendo la causa de enfermedades cardiovasculares, accidentes cerebrovasculares, ataques al corazón y discapacidad. Además, el colesterol alto interviene en la aparición y mantenimiento de enfermedades crónicas como la obesidad y la hipertensión. Diferentes estudios (50) han demostrado que los jóvenes universitarios presentan riesgo lipídico cuando los valores son limítrofes, es decir, la prueba de hipercolesterolemia sobrepasa lo deseable: menos de $200 \mathrm{mg} / \mathrm{dL}$, a un límite alto: 200 - $239 \mathrm{mg} / \mathrm{dL}$, o presenta un valor alto: igual o mayor a $240 \mathrm{mg} / \mathrm{dL}$. Cuando ocurre esta situación es necesario implementar medidas preventivas dirigidas a promover una cultura de hábitos alimenticios saludables, y una mayor actividad física, constituyéndose en dos de las principales alternativas para la prevención de la obesidad. Los niveles elevados de colesterol en etapas tempranas de la vida juegan un rol fundamental en el desarrollo de ateroesclerosis del adulto. Es necesario recalcar, que en este estudio se encontró que el $80 \%$ de los estudiantes desconoce el nivel de triglicéridos en la sangre, lo que alerta sobre un riesgo para su salud. Resultado que se refuerza en diferentes investigaciones (50) en donde se ha encontrado que el grupo etáreo entre 20 a 29 años presenta mayor frecuencia de hipertrigliceridemia, y esto se agrava a medida que aumenta la edad apareciendo las dislipidemias.

Del mismo modo se evidencia que el peso corporal y la distribución de la grasa corporal influyen en los niveles de triglicéridos (50); de manera semejante influyen: los malos hábitos alimenticios, el sedentarismo, el sobrepeso, la obesidad, el incremento en el consumo de productos con alto contenido de calorías, grasas trans y carbohidratos. Todas estas prácticas alteran los niveles de lípidos plasmáticos de los universitarios. Algo semejante ocurre con las enfermedades como el hipertiroidismo, la diabetes, el hiperinsulinismo, las enfermedades renales y las hepáticas, la menopausia, algunos fármacos y factores genéticos, siendo factores que incrementan los triglicéridos (51).

Por último, casi la mitad de los estudiantes no comparte el tiempo con pares, como amigos cercanos, familia o compañeros de curso. Contar con compañeros, amigos, familiares tiene una relación directa con el grado de estrés académico del estudiante, la salud mental y el rendimiento académico (52).

\section{Conclusiones}

- El acceso a la universidad de los jóvenes puede considerarse como un momento crucial en el que se modifican hábitos de vida, que con frecuencia se mantiene en la edad adulta con el consiguiente efecto (riesgo o beneficio) sobre la salud. Una vez realizado este estudio, se descubren los siguientes hallazgos, que evidencian la necesidad de desarrollar programas para promover estilos de vida saludables.

- Los comportamientos en salud de los estudiantes permitieron identificar que es necesario im- 
pulsar áreas tales como: la actividad física, la alimentación saludable, el no consumo de cigarrillo y alcohol, el manejo adecuado de la ansiedad y la angustia, la conformación de redes de apoyo, el autocuidado de la salud y mejorar la percepción del peso, entendida esta última como la relación existente el mayor peso y su incidencia en un mayor riesgo de desarrollar diferentes enfermedades, más no como un problema estético.

- Como se ha demostrado, entre todas las patologías y riesgos para la salud y la calidad de vida, que tienen mayor impacto en la salud pública se relacionan con los factores de riesgo cardiovascular. Según los hallazgos encontrados en la encuesta y el hecho de que el $63 \%$ de los estudiantes de primer semestre que ingresan a la universidad estén en riesgo nutricional, siendo menores de 20 años, presentan una mayor probabilidad de riesgo; igualmente, visibilizan la importancia de educar y crear hábitos de vida saludable, los cuales se relacionan con la reducción del riesgo de padecer enfermedades crónicas no trasmisibles.

- Así mismo, se sabe que impactan positivamente sobre el estudiante el ejercicio físico, la alimentación saludable, la red social, el adecuado manejo de estresores, lo que se ve reflejado en la esfera del rendimiento académico, disminuyéndose la deserción escolar causada por enfermedades, adicciones y presión social (estereotipos sociales).

\section{Conflicto de intereses}

Los autores declaran no tener ningun conflicto de intereses

\section{Referencias Bibliográficas}

1. Organización Mundial de la Salud. Promoción de la salud. Glosario. [Internet].1998 [consultado 12 de octubre 2019]. Disponible en: https://apps.who.int/iris/bitstream/handle/10665/67246/WHO_HPR_HEP_98.1_spa. pdf;jsessionid=ED1938CC-910D89545E906EA4CDD619CB? sequence $=1$

2. Organización Mundial de la Salud. Informe sobre la situación mundial de las enfermedades no trasmisibles. [Internet] 2014[consultado 12 de octubre 2019]; 1-16. Disponible en: https://apps.who.int/iris/bitstream/handle/10665/149296/WHO NMH_NVI_15.1 spa.pdf;jsessionid=AC7337B52995AE6A214D$\underline{67552 \mathrm{D} 2 \mathrm{EA} 546 ? \text { sequence }=1}$

3. Organización Mundial de la Salud. enfermedades no trasmisibles. [Internet] 2018[consultado el 12 de octubre 2019] Disponible en: http://www.who.int/mediacentre/factsheets/fs355/es/

4. Organización Mundial de la Salud. Diseño de servicios de salud acorde con esquemas de protección social. [Internet] 2017 [consultado 12 de octubre 2019] Disponible en: http://www.paho.org/chi/index.php?op$\underline{\text { tion }=\text { com content } \& \text { view }=\text { article } \& i d=128 \text { :enfermedades-no-transmisibles } \& \text { Itemid }=213}$

5. Ministerio de salud y protección social. Análisis de situación de salud (ASIS) Colombia. Dirección de Epidemiologia y demografía. Bogotá, (Colombia); [Internet]. 2016 [consultado 12 de octubre 2019]. Disponible en: https://www.minsalud.gov.co/sites/rid/Lists/BibliotecaDigital/RIDE/VS/ED/PSP/asis-colombia-2016.pdf

6. Organización Mundial de la Salud. Los jóvenes y los riesgos sanitarios. [Internet] 2018 [consultado 12 de octubre 2019] Disponible en: https://www.who.int/es/news-room/fact-sheets/detail/adolescents-health-risks-and-solutions

7. Cabrera-Díaz E, Aztaisa-Arías GM, Charry-Méndez S. Factores de riesgo para enfermedades no trasmisibles en universitarios: validación de contenido de un cuestionario. Tempuspsi. [Internet]. 2020 [consultado 10 de Marzo 2020] 4(1); 67-87. Disponible en: https://revistasum.umanizales.edu.co/ojs/index.php/tempuspsi/ article/view/3378/6459 
8. Práxedes A, Sevil J, Moreno A, Del villar F, García-González, L. Niveles de actividad Física en estudiantes universitarios: diferencias en función del género, la edad y los estados de cambio. Revista iberoamericana de psicología del Ejercicio y el Deporte. [Internet]. 2016 [consultado 14 de octubre 2019] 11(1); 123-132. Disponible en: https://dialnet.unirioja.es/servlet/articulo?codigo $=5287993$

9. Molina-Arellano CX, Andrade-Salas HM. El ejercicio para jóvenes universitarios. Rev. UNIMAR [Internet]. 2017 [consultado 14 de octubre de 2019]; 34(1). Disponible en: http://editorial.umariana.edu.co/revistas/index.php/unimar/article/view/1142

10. Cambronero-Resta M, Blasco-Mira JE, Chiner-Sanz E, Luca-Cuevas AG. Motivos de participación de los estudiantes universitarios en actividades físicos-deportivas. Revista iberoamericana de Psicología y el deporte. [Internet]. 2015 [consultado 14 de Octubre 2019] 10(2); 179-186. Disponible en: https://dialnet.unirioja.es/ servlet/articulo? codigo $=5083104$

11. Leiva AM, Martínez MA, Cristi-Montero C, Salas C, Ramírez-Campillo R, Díaz Martínez X, Aguilar-Farías N, Celis-Moreno C. El sedentarismo se asocia a un incremento de factores de riesgo cardiovascular y metabólico independiente de los niveles de actividad física. Rev Med Chile. [Internet]. 2017 [consultado 14 de octubre 2019] 14 (5); 458-467. Disponible en: https://scielo.conicyt.cl/pdf/rmc/v145n4/art06.pdf

12. Caro-Freile AI, Rebolledo-Cobos RC. Determinantes para la Práctica de Actividad Física en Estudiantes Universitarios. Duazary [Internet]. 2017 [consultado 11 de marzo de 2019]; 14(2):204-11. Disponible en: https:// revistas.unimagdalena.edu.co/index.php/duazary/article/view/1969

13. Castañeda-Vázquez C, Campos-Mesa MC, Del Castillo Andrés O. Actividad física y percepción de salud de los estudiantes universitarios. Rev. Fac. Med. [Internet]. 2016 [consultado 11 de marzo de 2019]; 64(2): $277-$ 284. Disponible en: http://www.scielo.org.co/pdf/rfmun/v64n2/v64n2a13.pdf

14. 14. Cabrera-Díaz E, Charry-Méndez S. Promoción del autocuidado de la salud en estudiantes universitarios. Proyección social [Internet] 2020 [consultando 29 de abril 2021]; 4(1):54-62. Disponible en: https://journalusco.edu.co/index.php/rps/article/view/3028/4089

15. Ruiz-Moreno E, Del Pozzo S, Valero-Gaspar T, Ávila Torres J, Varela-Moreiras, G. Estudio De Hábitos Alimentarios Y Estilos De Vida De Los Universitarios Españoles. Patrón de consumo de bebidas fermentadas. Fundación Española de la Nutrición (FEN), Universidad CEU San Pablo (Madrid). [Internet] 2016 [Consultado el 20 de diciembre del 2019]. Disponible en: https://www.fen.org.es/storage/app/media/imgPublicaciones/30092014131915.pdf

16. Rangel-Caballero LG, Rojas-Sánchez LZ, Gamboa-Delgado EM. Actividad física y composición corporal en estudiantes universitarios de cultura física, deporte y recreación. Rev. Univ. Ind. Santander Salud. [Internet]. 2015 [consultado 11 de marzo de 2019]; 47(3): 281-289. Disponible en: https://revistas.uis.edu.co/index.php/ revistasaluduis/article/view/5134/5474

17. Elveny-Laguado J, Gómez-Díaz MP. Estilos de vida saludable en estudiantes de enfermería en la Universidad Cooperativa de Colombia. Hacia promoc. salud. [Internet]. 2014 [consultado 11 de marzo de 2019]; 19 (1): 68 - 83. Disponible en: http://www.scielo.org.co/pdf/hpsal/v19n1/v19n1a06.pdf

18. Salazar-Blandón DA, Alzate-Yepes T, Múnera-Gaviria HA, Pastor-Durango MP. Sobrepeso, obesidad y factores de riesgo: un modelo explicativo para estudiantes de Nutrición y Dietética de una universidad pública de Medellín, Colombia. Perspect Nut Hum [Internet]. 2020 [citado 29 de abril de 2021];22(1):47-9. Disponible en: https://revistas.udea.edu.co/index.php/nutricion/article/view/339342

19. Pérez-Ugidos G, Laíño F, Zelarayán J, Márquez S. Actividad física y hábitos de salud en estudiantes universitarios Argentinos. Rev. Nutr Hosp. [Internet] 2014 [consultado 15 abril 2019]; 30(4):896-904. Disponible en: http://scielo.isciii.es/scielo.php?script=sci arttext\&pid=S0212-16112014001100026 
Scientific Journal of Nursing

20. Cardona-Torres LM, Centeno-Reséndiz JV, Álvarez-Hernández A, Medina-López E. Calidad de vida relacionada con la salud: de los adolescentes obesos, no obesos y sus padres. Rev Enferm Inst Mex Seguro Soc. [Internet]. 2018 [consultado 15 de abril del 2019]; 26(2): 121-128. Disponible en: https://www.medigraphic. com/pdfs/enfermeriaimss/eim-2018/eim182i.pdf

21. Ulloa-López MP, Vásquez-Amores A. Prevalencia del bajo rendimiento académico universitario y factores asociados en la carrera de medicina. [Tesis pregrado en internet]. [Cuenca]: Universidad de Cuenca; 2015. [consultado 15 de abril 2019]. Disponible en: http://dspace.ucuenca.edu.ec/bitstream/123456789/22430/1/ tesis.pdf

22. Castejón-Martínez MA, Berengui GR, Garcés de los Fayos Ruiz, EJ. Relación del índice de masa corporal, percepción de peso y variables relacionadas con los trastornos de la conducta alimentaria en estudiantes universitarios. Nutr. Clínica y Dietética Hospitalaria. [internet]. 2016 [consultado 12 de Mayo 2019]; 36 (1): 54-63. Disponible en: https://revista.nutricion.org/PDF/361 castejon.pdf

23. Duno M, Acosta E. Percepción de la imagen corporal en adolescentes universitarios. Rev. Chil. Nutr. [Internet]. 2019 [consultado 25 noviembre 2019]; 46(5). Disponible en: https://scielo.conicyt.cl/scielo.php?script=sci arttext\&pid $=$ S0717-75182019000500545

24. Cheesman-Mazariegos S, Suárez-Lugo N. Tabaquismo en estudiantes de Medicina de la Universidad de San Carlos Guatemala. Rev. cubana de Salud Pública. [Internet]. 2015 [consultado 12 de mayo 2019]; 41(1):1832. Disponible en: https://www.medigraphic.com/cgi-bin/new/resumen.cgi?IDARTICULO=55508

25. Mantilla-Toloza SC, Villamizar CE, Peltzer K. Consumo de alcohol, tabaquismo y características sociodemográficas en estudiantes universitarios. Rev. Univ. salud. [Internet]. 2016 [consultado 12 de mayo 2019]; 18(1):7-15. Disponible en: https://revistas.udenar.edu.co/index.php/usalud/article/view/2718

26. Becerra S. Descripción de las conductas de salud en un grupo de estudiantes universitarios de Lima. Rev. de psicología. [Internet]. 2016 [consultado 12 de mayo 2019]; 34(2):242-259. Disponible en: http://revistas. pucp.edu.pe/index.php/psicologia/article/view/14938/15473

27. Barreto-Niño AN, Bonilla-Reyes PA, Calderón-Bonilla JF, Cantillo-Aviléz MA, Viña-Lopera JA, Quitian-Reyes $\mathrm{H}$. factores asociados al inicio del hábito de fumar en estudiantes universitarios en Bogotá, Colombia. Univ. Med. [Internet]. 2018 [consultado 12 de mayo de 2019]; 59 (2): 2-7. Disponible en: http://www. scielo.org.co/pdf/unmed/v59n2/0041-9095-unmed-59-02-00017.pdf

28. Ariza-Delgado J. Factores relacionados al consumo de tabaco y la prevalencia de este hábito en los estudiantes de enfermería de la pontificia universidad javeriana durante el segundo semestre del año 2016. [Tesis pregrado]. Bogotá. Pontificia Universidad Javeriana Facultad de Enfermería; 2016.

29. Betancourt-Zambrano S, Tacán-Bastidas L, Córdoba-Paz E. Consumo de alcohol en estudiantes universitarios colombianos. Rev Univ. Salud. [Internet]. 2017 [consultado 12 de mayo de 2019]; 19(1):37-50. Disponible en: https://revistas.udenar.edu.co/index.php/usalud/article/view/2861

30. González-Ospina A, Rodríguez-Restrepo A, Grajales-Román MM, Espinosa-Chirivi Y, Martínez-Gómez ML, Agudelo-Suárez AA. Frecuencia y factores asociados al consumo de alcohol, cigarrillo y sustancias psicoactivas, en estudiantes de una Universidad Pública en Colombia: Un estudio mixto. Rev. nac. odontol. [Internet]. 2020 [consultado 12 de agosto de 2020]; 16(1):1-19. Disponible en: https://revistas.ucc.edu.co/index.php/od/ article/view/363

31. Montaño-Sinisterra MR, Guarín AD, Gómez-Millán MA. Consumo de alcohol en estudiantes de la Universidad de San Buenaventura sede Bogotá. [Tesis pregrado en internet]. [Bogotá]. Universidad de San Buenaventura sede Bogotá. 2011. [Consultado el 12 de noviembre de 2019]. Disponible en: http://bibliotecadigital.usb. edu.co/bitstream/10819/723/1/Consumo_alcohol estudiantes_Monta\%C3\%B1o_2011.pdf 
Scientific Journal of Nursing

32. Cerquera I, Córdoba S, Garzón A. Prevalencia del consumo de bebidas alcohólicas en adolescentes y factores asociados. Cina Research. [internet]. 2019 [consultado 12 de agosto de 2019]; 3(1):70-77. Disponible en: https://journals.uninavarra.edu.co/index.php/cinaresearch/article/view/179

33. Andrade-Obregón LM, Ramírez-Ortiz DC, Muñoz ML. Relación entre el consumo de alcohol y el rendimiento académico en estudiantes de dos universidades de Bogotá. [Tesis pregrado en internet]. [Bogotá]. Pontificia Universidad Javeriana, Facultad de Psicología, [consultado 12 de noviembre del 2017]. Recuperadoapartirde: https://repository.javeriana.edu.co/bitstream/handle/10554/7980/tesis48.pdf? sequence=1\&isAllowed=y

34. Medline Plus-información de salud de la Biblioteca Nacional de Mediclina. Alcoholismo y abuso de álcool [Internet]. 2016. [consultado 12 de Diciembre del 2017]. Disponible en: https://medlineplus.gov/spanish/ alcoholismandalcoholabuse.html

35. Arévalo-García E, Castillo-Jimenez DA, Cepeda I, López-Pacheco J, Pachecho-López R. Anxiety and depression in university students: relationship with academic performance. Interdiscip J Epidemiol Pub Health [Internet]. 2020 [consultado el 12 septiembre del 2020];2(1):e-022. Dsiponible en: https://revistas.unilibre. edu.co/index.php/iJEPH/article/view/5342

36. Castillo-Pimienta C, Chacón-de-la-Cruz T, Díaz-Véliz G. Ansiedad y fuentes de estrés académico en estudiantes de carreras de la salud. Investigación en educación médica. [Internet]. 2016 [consultado 12 de agosto del 2019]; 5 (20): 230-237. Disponible en: https://www.sciencedirect.com/science/article/pii/S2007505716000491

37. Bonifacio-Vilela M, Caro-Salazar CA, Domínguez-Lara SA. Prevalencia de ansiedad ante exámenes en una muestra de estudiantes universitarios de lima metropolitana. Revista de psicología. [Internet]. 2016 [consultado 12 de agosto del 2019]; 6(2): 47-56. Disponible en: https://revistas.ucsp.edu.pe/index.php/psicologia/ article/view/155

38. Solera-Sánchez A, Gamero-Lluna A. Hábitos saludables en universitarios de ciencias de la salud y de otras ramas de conocimiento: un estudio comparativo. Rev Esp Nutr Diet. [Internet]. 2019 [consultado 12 de agosto de 2019]; 23(4):2-38. Disponible en: https://renhyd.org/index.php/renhyd/article/view/762/549

39. Becerra F, Pinzón G, Vargas M. Food practices of a group of college students and the perceived difficulties for healthy eating. Rev.Nutr. Humana, Facultad de Medicina. [Internet]. 2015 [consultado 12 de agosto de 2019]; 63; 57-463. Disponible en: http://www.scielo.org.co/scielo.php?script=sci_arttext\&pid=S0120-00112015000300013

40. Rojas, D. Percepción de alimentación saludable, hábitos alimentarios estado nutricional y práctica de actividad física en población de 9-11 años del colegio cedid ciudad bolívar, Bogotá. [Tesis de Pregrado en internet]. [Bogotá]: Pontificia Universidad Javeriana. Bogotá. Colombia. 2011. [Consultado el 10 de diciembre 2017]. Disponible en: http://www.javeriana.edu.co/biblos/tesis/ciencias/tesis704.pdf

41. Villaquíran AF, Cuero Vivas PA, Cerón GM, Ordoñez A, Jácome S. Características antropométricas, hábitos nutricionales, actividad física y consumo de alcohol en estudiantes universitarios. Salud UIS. [Internet]. 2020 [consultado 23 agosto de 2020]; 52(2):111-120. Disponible en: https://revistas.uis.edu.co/index.php/revistasaluduis/article/view/10642/10448

42. Rodríguez-Leyton M, Sánchez-Majana L. Consumo de frutas y verduras: Beneficios y retos. Alimentos hoy. [Internet] 2017 [consultado 29 abril de 2021]; 25(42): 34-55. Disponible en: https://alimentoshoy.acta.org.co/ index.php/hoy/article/view/457

43. Organización Mundial de la Salud. Alimentación Sana. [Internet]. 2015 [consultado 12 diciembre 2019]. Disponible en: http://www.who.int/mediacentre/factsheets/fs394/es/

44. Durán S, Valdés P, Godoy A, Herrera T. Consumo de frutas y su asociación con el estado nutricional en estudiantes universitarios chilenos de la carrera de educación física. Rev. Nutr. Hospitalaria. [Internet]. 2015 
Scientific Journal of Nursing

[consultado 12 de agosto 2019]; 31(5):2247-2252. Disponible en: http://scielo.isciii.es/scielo.php?script=sci arttext\&pid $=$ S0212-16112015000500045

45. Huamancayo Espíritu A, Pérez Cárdenas L. Factores asociados al consumo de fruta y verdura en alumnos de medicina de una universidad peruana. Revista peruana de investigación en salud. [Internet]. 2019 [consultado 13 de diciembre 2019]; 3(4). Disponible en: http://portal.amelica.org/ameli/jatsRepo/100/100857002/html/ index.html

46. De Piero A, Bassett N, Rossi A, Sammán N. Tendencia en el consumo de alimentos de estudiantes universitarios. Rev. Nutr Hosp. [Internet].2015 [consultado 13 de diciembre 2019]; 31(4):1824-1831. Disponible en: http://scielo.isciii.es/scielo.php?script $=$ sci arttext\&pid=S0212-16112015000400049

47. ICBF Profamilia ENSIN. Encuesta Nacional de la situación Nutricional. [internet]. 2015 [consultado 12 de diciembre 2019]. Disponible en: www.icbf.gov.co/bienestar/nutricion/encuesta-nacional-situacion-nutricion$\underline{\text { al. }}$

48. Fundación Sochicar Sociedad Chilena de cardiología y cirugía cardiovascular. La importancia del chequeo Médico. [Internet]. 2016 [consultado 12 de diciembre 2019]. Disponible en: http://portal.sochicar.cl/Mujeres-en-Rojo/Mujeres-en-Rojo/la-importancia-del-chequeo-medico.html

49. Organización Mundial de la Salud. El colesterol alto, un problema mal controlado. [Internet]. 2016 [consultado 27 de septiembre 2019]. Disponible en: http://www.who.int/mediacentre/factsheets/fs317/es/

50. Morales G, Guillen Grima SM, Belmar C, Schifferli I, Muñoz A, Soto A. Factores de riesgo cardiovascular en universitarios de primer y tercer año. Rev. Med. Chile. [Internet]. 2017 [consultado 12 de diciembre 2019]; 145:299-308. Disponible en: https://scielo.conicyt.cl/pdf/rmc/v145n3/art03.pdf

51. Carranza-Madrigal J. Triglicéridos y riesgo cardiovascular. Med Int Méx. [Internet]. 2017 [consultado 29 de abril 2021]; 33(4):511-514. Disponible en: https://www.medigraphic.com/pdfs/medintmex/mim-2017/ mim174i.pdf

52. Obregón-Morales B, Montalván-Romero JC, Segama Fabian E, Dámaso Mata B, Panduro Correa V, Arteaga Livias K. Factores asociados a la depresión en estudiantes de medicina de una universidad peruana. Educ Med Super [Internet]. 2020 [consultado 29 abril 2021]; 34(2): e1881. Disponible en: http://scielo.sld.cu/scielo. php?script $=$ sci arttext\&pid=S0864-21412020000200013\&lng=es 